\title{
Gaussian Quadrature of Some Integrals Involving Airy Functions
}

\author{
By J. A. Riley and C. Billings
}

1. Summary. The integrals

$$
\begin{array}{rlll}
\int_{0}^{\infty} x^{\lambda} \frac{A}{F^{2}} d x, & \int_{0}^{\infty} x^{\lambda} \frac{A^{2}}{F^{2}} d x, & \int_{0}^{\infty} x^{\lambda} \frac{A B}{F^{2}} d x, & \int_{0}^{\infty} x^{\lambda} \frac{B}{F^{2}} d x, \\
\int_{0}^{\infty} x^{\lambda} \frac{A^{\prime}}{F^{\prime 2}} d x, & \int_{0}^{\infty} x^{\lambda} \frac{A^{\prime 2}}{F^{\prime 2}} d x, & \int_{0}^{\infty} x^{\lambda} \frac{A^{\prime} B^{\prime}}{F^{\prime 2}} d x, & \int_{0}^{\infty} x^{\lambda} \frac{B^{\prime}}{F^{\prime 2}} d x,
\end{array}
$$

(in which $A, B$ are the Airy functions $A i, B i$, and in which $A^{\prime}=d A / d x, B^{\prime}=d B / d x$, $F^{2}=A^{2}+B^{2},{F^{\prime}}^{2}=A^{\prime 2}+B^{\prime 2}$ ) are the coefficients in certain power series arising in the diffraction theory of electromagnetic waves. The values of these integrals were computed for $\lambda=0,1, \cdots, 20$ by dividing the interval of integration into four closed subintervals $(0,8),(8,16),(16,24),(24, \infty)$ and carrying out the integration over each interval separately. The integrations in each of the first three intervals were performed by using a 27-abscissa Gaussian quadrature scheme. The integration over $(24, \infty)$ was effected by exact integration of an asymptotic form for the integrands. The values of the integrals are shown in Table 1. Although an exact estimate of the total error was not obtained, it seems reasonable that the results are correct in most cases to five significant figures. An independent check on the accuracy has been provided by certain calculations made at the Electromagnetic Radiation Laboratory, Air Force Cambridge Research Center, in which our results were used.

2. The Integration Scheme. The integrals (1) have the general form

$$
I(\lambda)=\int_{0}^{\infty} x^{\lambda} f(x) d x .
$$

An examination of the values of the integrands will show that they have a skew 'bell-shape' rising from zero at $x=0$ to a maximum point somewhere in the interval $(0,8)$, and thereafter decaying quite rapidly to zero. On the basis of this behavior, and for convenience, the interval of integration is subdivided into four intervals: $(0,8),(8,16),(16,24),(24, \infty)$. In the course of the numerical evaluation, it is easy to check whether sufficient accuracy is obtained by retaining only the first integral, or the first two, etc. Thus we write:

$$
I(\lambda)=\int_{0}^{8} x^{\lambda} f(x) d x+\int_{8}^{16} x^{\lambda} f(x) d x+\int_{16}^{24} x^{\lambda} f(x) d x+\int_{24}^{\infty} x^{\lambda} f(x) d x .
$$

Received August 14, 1958. The work reported here was done under a contract with the Electromagnetic Radiation Laboratory, Air Force Cambridge Research Center. The Gaussian abscissas in Table II were computed by Murray Sherry using the ERD Applied Mathematics Group's computer. 
TABLE 1. Values of the Integrals

\begin{tabular}{|c|c|c|c|c|}
\hline$\lambda$ & $\int_{0}^{\infty} x^{\lambda} \frac{A}{F^{2}} d x$ & $\int_{0}^{\infty} x^{\lambda} \frac{A^{2}}{F^{2}} d x$ & $\int_{0}^{\infty} x^{\lambda} \frac{A B}{F^{2}} d x$ & $\int_{0}^{\infty} x^{\lambda} \frac{B}{F^{2}} d x$ \\
\hline 0 & $3.6022 \times 10^{-1}$ & $9.2361 \times 10^{-2}$ & $3.0900 \times 10^{-1}$ & 1.8134 \\
\hline 1 & $1.5318 \times 10^{-1}$ & $2.9547 \times 10^{-2}$ & $1.7071 \times 10^{-1}$ & 1.8013 \\
\hline 2 & $1.1372 \times 10^{-1}$ & $1.6929 \times 10^{-2}$ & $1.4551 \times 10^{-1}$ & 2.9080 \\
\hline 3 & $1.1448 \times 10^{-1}$ & $1.3333 \times 10^{-2}$ & $1.9582 \times 10^{-1}$ & 6.1454 \\
\hline 4 & $1.4091 \times 10^{-1}$ & $1.3014 \times 10^{-2}$ & $3.1323 \times 10^{-1}$ & $1.5673 \times 10$ \\
\hline 5 & $2.0194 \times 10^{-1}$ & $1.4915 \times 10^{-2}$ & $5.7396 \times 10^{-1}$ & $4.6231 \times 10$ \\
\hline 6 & $3.2683 \times 10^{-1}$ & $1.9427 \times 10^{-2}$ & 1.1815 & $1.5361 \times 10^{2}$ \\
\hline 7 & $5.8546 \times 10^{-1}$ & $2.8137 \times 10^{-2}$ & 2.6921 & $5.6466 \times 10^{2}$ \\
\hline 8 & 1.4433 & $4.4623 \times 10^{-2}$ & 6.7053 & $2.2662 \times 10^{3}$ \\
\hline 9 & 2.4148 & $7.6606 \times 10^{-2}$ & $1.8073 \times 10$ & $9.8304 \times 10^{3}$ \\
\hline 10 & 5.4566 & $1.4111 \times 10^{-1}$ & $5.2283 \times 10$ & $4.5719 \times 10^{4}$ \\
\hline 11 & $1.3117 \times 10$ & $2.7692 \times 10^{-1}$ & $1.6124 \times 10^{2}$ & $2.2648 \times 10^{5}$ \\
\hline 12 & $3.3366 \times 10$ & $5.7576 \times 10^{-1}$ & $5.2719 \times 10^{2}$ & $4.8862 \times 10^{6}$ \\
\hline 13 & $8.9412 \times 10$ & 1.2622 & $1.8189 \times 10^{3}$ & $6.5785 \times 10^{6}$ \\
\hline 14 & $2.5146 \times 10^{2}$ & 2.9062 & $6.5962 \times 10^{3}$ & $3.8247 \times 10^{7}$ \\
\hline 15 & $7.3980 \times 10^{2}$ & 7.0037 & $2.5077 \times 10^{4}$ & $2.3280 \times 10^{8}$ \\
\hline 16 & $2.2704 \times 10^{3}$ & $1.7614 \times 10$ & $9.9395 \times 10^{4}$ & $1.4791 \times 10^{9}$ \\
\hline 17 & $7.2503 \times 10^{3}$ & $4.6113 \times 10$ & $4.1080 \times 10^{5}$ & $9.7850 \times 10^{9}$ \\
\hline 18 & $2.4039 \times 10^{4}$ & $1.2537 \times 10^{2}$ & $1.7634 \times 10^{6}$ & $6.7240 \times 10^{10}$ \\
\hline 19 & $8.2588 \times 10^{4}$ & $3.5333 \times 10^{2}$ & $7.8520 \times 10^{6}$ & $4.7898 \times 10^{11}$ \\
\hline 20 & $2.9349 \times 10^{5}$ & $1.0302 \times 10^{3}$ & $3.3990 \times 10^{7}$ & $3.5306 \times 10^{12}$ \\
\hline$\lambda$ & $\int_{0}^{\infty} x^{\lambda} \frac{A^{\prime}}{F^{\prime 2}} d x$ & $\int_{0}^{\infty} x^{\lambda} \frac{A^{\prime 2}}{F^{\prime 2}} d x$ & $\int_{0}^{\infty} x^{\lambda} \frac{A^{\prime} B^{\prime}}{F^{\prime 2}} d x$ & $\int_{0}^{\infty} x^{\lambda} \frac{B^{\prime}}{F^{\prime 2}} d x$ \\
\hline 0 & $6.6995 \times 10^{-1}$ & $1.5095 \times 10^{-1}$ & $4.0149 \times 10^{-1}$ & 2.1981 \\
\hline 1 & $2.9569 \times 10^{-1}$ & $5.8235 \times 10^{-2}$ & $2.3627 \times 10^{-1}$ & 1.8358 \\
\hline 2 & $2.0787 \times 10^{-1}$ & $3.5690 \times 10^{-2}$ & $2.2253 \times 10^{-1}$ & 2.5158 \\
\hline 3 & $1.9019 \times 10^{-1}$ & $2.8304 \times 10^{-2}$ & $2.7450 \times 10^{-1}$ & 4.6331 \\
\hline 4 & $2.1044 \times 10^{-1}$ & $2.6958 \times 10^{-2}$ & $4.1160 \times 10^{-1}$ & $1.0577 \times 10$ \\
\hline 5 & $2.7098 \times 10^{-1}$ & $2.9673 \times 10^{-2}$ & $7.2041 \times 10^{-1}$ & $2.8549 \times 10$ \\
\hline 6 & $3.9631 \times 10^{-1}$ & $3.6859 \times 10^{-2}$ & 1.4332 & $8.8270 \times 10$ \\
\hline 7 & $6.4691 \times 10^{-1}$ & $5.0821 \times 10^{-2}$ & 3.1793 & $3.0570 \times 10^{2}$ \\
\hline 8 & 1.1649 & $7.6820 \times 10^{-2}$ & 7.7504 & $1.6652 \times 10^{3}$ \\
\hline 9 & 2.2771 & $1.2606 \times 10^{-1}$ & 2.0531 & $4.8440 \times 10^{3}$ \\
\hline 10 & 4.8106 & $2.2276 \times 10^{-1}$ & $5.8558 \times 10$ & $2.1679 \times 10^{4}$ \\
\hline 11 & $1.0911 \times 10$ & $4.2105 \times 10^{-1}$ & $1.7851 \times 10^{2}$ & $1.0378 \times 10^{5}$ \\
\hline 12 & $2.6334 \times 10$ & $8.4640 \times 10^{-1}$ & $5.7806 \times 10^{2}$ & $5.2801 \times 10^{5}$ \\
\hline 13 & $6.7332 \times 10$ & 1.8006 & $1.9785 \times 10^{3}$ & $2.8408 \times 10^{6}$ \\
\hline 14 & $1.8155 \times 10^{2}$ & 4.0365 & $7.1263 \times 10^{3}$ & $1.6092 \times 10^{7}$ \\
\hline 15 & $5.1394 \times 10^{2}$ & 9.4996 & $2.6914 \times 10^{4}$ & $9.5617 \times 10^{7}$ \\
\hline 16 & $1.5244 \times 10^{3}$ & $2.3394 \times 10$ & $1.0624 \times 10^{5}$ & $5.9404 \times 10^{8}$ \\
\hline 17 & $4.7169 \times 10^{3}$ & $6.0109 \times 10$ & $4.3707 \times 10^{5}$ & $3.8494 \times 10^{9}$ \\
\hline 18 & $1.5193 \times 10^{4}$ & $1.6073 \times 10^{2}$ & $1.8714 \times 10^{6}$ & $2.5925 \times 10^{10}$ \\
\hline 19 & $5.0821 \times 10^{4}$ & $4.4628 \times 10^{2}$ & $8.2973 \times 10^{6}$ & $1.8127 \times 10^{11}$ \\
\hline 20 & $1.7618 \times 10^{5}$ & $1.2840 \times 10^{3}$ & $3.8129 \times 10^{7}$ & $1.3127 \times 10^{12}$ \\
\hline
\end{tabular}


Transforming the first three integrals in (3) to the interval $[-1,1]$ we obtain:

$$
I(\lambda)=I_{0}^{8}+I_{8}^{16}+I_{16}^{24}+\int_{24}^{\infty} x^{\lambda}(x) d x
$$

in which

$$
\left\{\begin{array}{l}
I_{0}^{8}=4^{\lambda+1} \int_{-1}^{1}(1+y)^{\lambda} f(4[1+y]) d y, \\
I_{8}^{16}=4 \int_{-1}^{1}(8+4[1+y])^{\lambda} f(8+4[1+y]) d y, \\
I_{16}^{24}=4 \int_{-1}^{1}(16+4[1+y])^{\lambda} f(16+4[1+y]) d y .
\end{array}\right.
$$

The Gaussian quadrature formula with $n$ abscissas, and weight function 1 gives (cf $[1])$ :

$$
\begin{aligned}
& I_{0}^{8}=4^{\lambda+1} \sum_{j=1}^{n} H_{j}\left(1+a_{j}\right)^{\lambda} f\left(4\left[1+a_{j}\right]\right), \\
& I_{8}^{16}=4 \sum_{j=1}^{n} H_{j}\left(8+4\left[1+a_{j}\right]\right)^{\lambda} f\left(8+4\left[1+a_{j}\right]\right), \\
& I_{16}^{24}=4 \sum_{j=1}^{n} H_{j}\left(16+4\left[1+a_{j}\right]\right)^{\lambda} f\left(16+4\left[1+a_{j}\right]\right) .
\end{aligned}
$$

Here the $a_{j}$ are the roots of the Legendre polynomial, $P_{n}(x)$, of order $n$, and the $H_{j}$ are the corresponding weights. These weights are given by:

$$
H_{j}=\frac{2}{\left(1-a_{j}^{2}\right)\left[P_{n}^{\prime}\left(a_{i}\right)\right]^{2}} ; \quad P_{n}^{\prime}(x)=\frac{d P_{n}}{d x} .
$$

The integration procedure then is to select a value of $n$, obtain the values of the abscissas $a_{j}$ and weights $H_{j}$, and evaluate the sums in (6).

3. Selection of $n$; Computation of the Integrals. Trial computations, using the tabulated values of the $a_{j}$ and $H_{j}[2]$ showed that a choice of $n=17$ gives the integrals, for $\lambda=0$, to five significant figures. For the higher values of $\lambda$, it is necessary to take a larger number of intermediate abscissas in order to retain the five significant figure accuracy. On the basis of the following, admittedly naive considerations, we chose $n=27$.

First of all, the degree of precision of the Gaussian quadrature formula with $n$ abscissas is $2 n-1$, i.e. the approximation gives exact results if the integrand is a polynomial of degree $2 n-1$ or less (On this point of [1].). Since $n=17$ gives "exact" five-figure accuracy for $\lambda=0$ then $f(4[1+y])$, in the interval $[-1,1]$ can be approximated to within a certain error by a polynomial of degree 33 . Then the functions $(1+y)^{\lambda} \cdot f(4[1+y])$ can be approximated by polynomials of degree $33+\lambda$. If these functions were actually polynomials of degree $33+\lambda$, the number of abscissas necessary for exact integration would be given by $2 n-1=33+\lambda$, or $n=17+\frac{1}{2} \lambda$. Since, for our cases, $\lambda$ ranges from 0 to 20 , we take $n=27$. We have not been able to obtain an estimate of the error in using 27 points for $\lambda>0$, but we feel from our experience with the computations, that the use of 27 abscissas retains the five figure accuracy of the results. 
TABLE 2. Gaussian Weights and Abscissas

$n=27$. Weight function $W(x)=1$.

\begin{tabular}{c|c}
\hline $\pm a_{j}$ & $H_{j}$ \\
\hline 0 & .114220849 \\
.113972586 & .113476364 \\
.226459365 & .111252467 \\
.335993904 & .107578263 \\
.441148252 & .102501615 \\
.540551565 & .096088705 \\
.632907972 & .088423136 \\
.717013550 & .079604846 \\
.791771639 & .069748802 \\
.856207908 & .058983516 \\
.909482321 & .047449392 \\
.970900558 & .035297282 \\
.996179263 & .022686214 \\
\hline
\end{tabular}

In order to obtain five significant figures in the integrals, it is necessary that the $I_{0}^{8}, I_{8}^{16}, I_{16}^{24}$ be correct to at least six significant figures. For this, because of roundoff errors, it is again necessary that the $H_{j}$ and the values of $f$ be correct to seven or eight significant figures, and this in turn means that the $a_{j}$ must be correct to eight or nine figures. Existing tables of the abscissas and weights do not contain the values with this accuracy. It is necessary then to compute these for our integrations. The $a_{j}$ are the roots of the Legendre polynomial

$$
P_{27}(x)=\sum_{j=0}^{13}(-1)^{j+1} 2^{-27}\left(\begin{array}{c}
27 \\
j+14
\end{array}\right)\left(\begin{array}{c}
2 j+28 \\
27
\end{array}\right) x^{2 j+1},
$$

and may be computed directly. The weights can be computed from (7).

Table 2 shows the weights and abscissas for $n=27$. The values are correct to nine decimal places.

The computation of the function values $f\left(4\left[1+a_{j}\right]\right)$, etc. were carried out using the British Association tables of the Airy functions [3]. In order to obtain these to eight figures it was necessary to use the second-order interpolation scheme suggested in the tables.

As noted below the value of the last integral,

$$
\int_{24}^{\infty} x^{\lambda} f(x) d x
$$

in (4) is negligible, in our cases, in comparison with the sum $I_{0}^{8}+I_{8}^{16}+I_{16}^{24}$. Consequently, the approximate integral is given by adding together the sums in the right side of (6). The results are shown in Table 1.

$$
\begin{aligned}
& \text { 4. Evaluation of } \int_{24}^{\infty} x^{\lambda} f(x) d x \text {. The contribution to the integral from the } \\
& \text { 'tail', } \\
& \qquad \int_{24}^{\infty} x^{\lambda} f(x) d x,
\end{aligned}
$$


was estimated by integrating an asymptotic approximation to the integrand. This asymptotic estimate is derived from the asymptotic forms of the Airy functions given in [3]. In view of the fact that even the values of $I_{16}^{24}$ were negligible in all cases when compared with the sums $I_{0}^{8}+I_{8}^{16}$, a satisfactory estimate of the integral

$$
\int_{24}^{\infty} x^{\lambda} f(x) d x
$$

is obtained by integration of the asymptotic form for $f$. The resulting integrals of these asymptotic expressions turn out to be incomplete gamma functions and may be evaluated with Pearson's tables [4]. The result is that the 'tails' made no contribution to the fifth figure of the sum $I_{0}^{8}+I_{0}{ }^{16}+I_{16}^{24}$, and are therefore neglected.

Parke Mathematical Laboratories, Inc.

Carlisle, Massachusetts

1. F. B. Hildebrand, Introduction to Numerical Analysis, McGraw-Hill, 1956, p. 312-367.

2. Z. Kopal, Numerical Analysis, Wiley, 1955, p. 523-525.

3. J. C. P. Miller, The Airy Integral, British Association Mathematical Tables, PartVolume B, University Press, Cambridge, 1946.

4. K. Pearson, Tables of the Incomplete $\Gamma$ Function, Cambridge University Press, 1951. 